\title{
CMEARTICLE
}

\section{Clinics in diagnostic imaging (197)}

Genevieve Jingwen $\underline{\operatorname{Tan}}^{1}$, MBBS, Niketa $\underline{\text { Chotai }}^{2}$, MBBS, FRCR, Ankit $\underline{\text { Tandon }}^{1}$, MBBS, FRCR

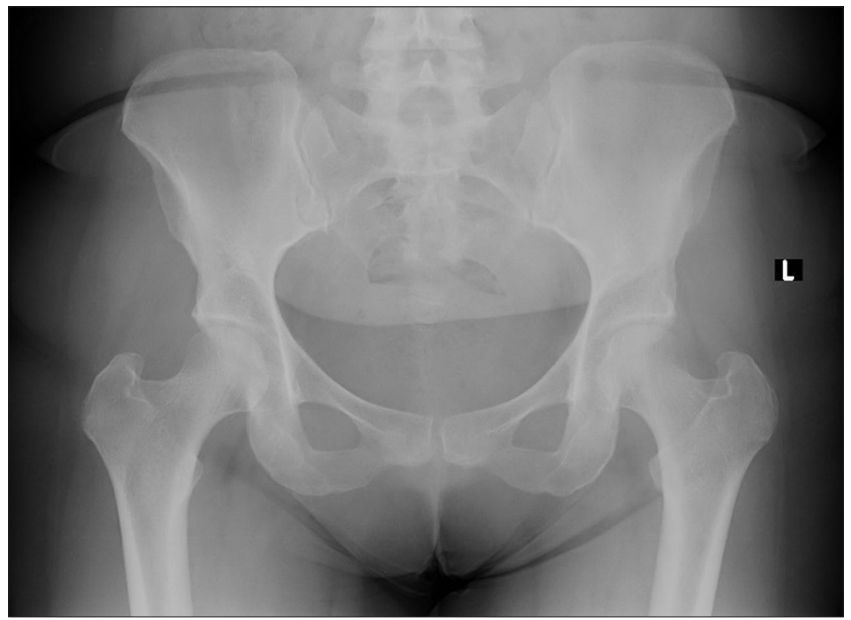

Fig. 1 Frontal radiograph of the pelvis.
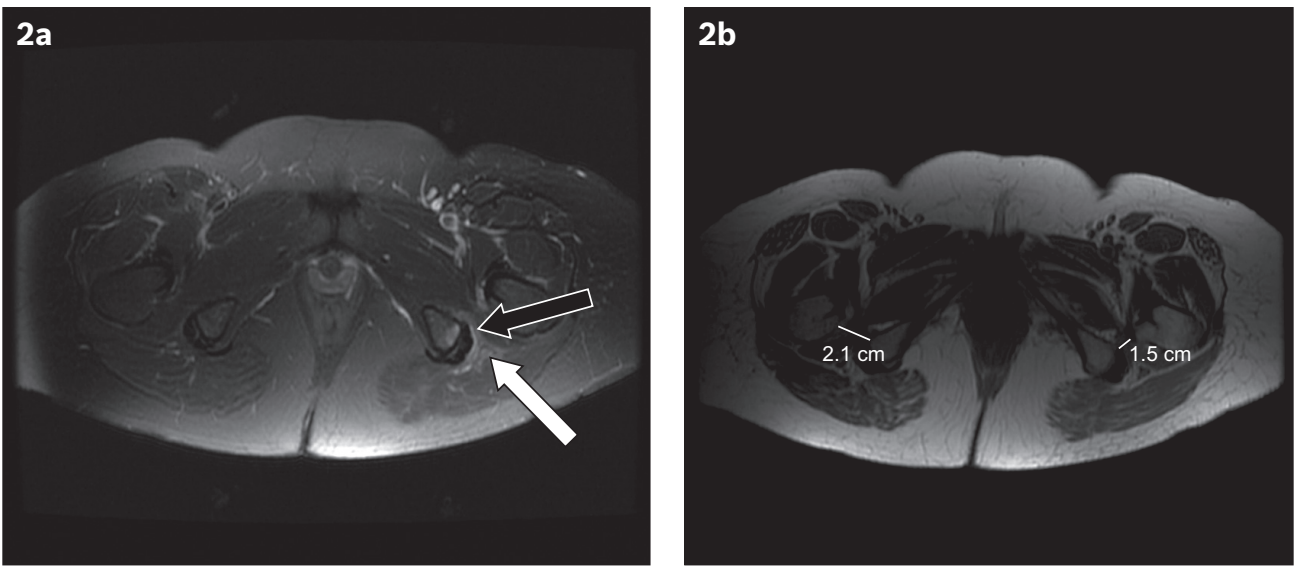

Fig. 2 (a) Axial T2-weighted fat suppression and (b) axial T1-weighted MR images of the pelvis.

\section{CASE PRESENTATION}

A 48-year-old woman presented with progressive left hip pain over six months, along with left lower limb weakness for two weeks. This had affected her gait and she experienced frequent falls. Physical examination revealed weakness of her left hip and knee extensor muscles as well as left ankle dorsiflexion. Magnetic resonance (MR) imaging of the left hip was performed. What do the pelvic radiograph (Fig. 1) and MR images (Fig. 2) of the left hip show? What is the diagnosis? 


\section{IMAGE INTERPRETATION}

Frontal radiograph of the pelvis (Fig. 1) shows no hip fracture or dislocation. No soft tissue abnormalities are detected. T2-weighted fat suppression MR imaging of the left hip (Fig. 2a) shows increased signal intensity involving the left quadratus femoris muscle (black arrow) and common hamstring tendons, along with mild left sciatic nerve thickening (white arrow). There is bone marrow oedema at the left ischial tuberosity at the site of attachment of the left common hamstring tendons. The left ischiofemoral space is narrower than the right, measuring $1.5 \mathrm{~cm}$ on the left and $2.1 \mathrm{~cm}$ on the right side (Fig. 2b). No fatty muscle atrophy is detected. No tendon avulsion or greater trochanteric bursitis is seen.

\section{DIAGNOSIS}

Left ischiofemoral impingement syndrome.

\section{CLINICAL COURSE}

The patient was started on a trial of conservative treatment with oral analgesia. Her symptoms persisted over the next 18 months. Eventually, her left hip pain progressed and she was offered decompressive surgery. She required a computed tomographyguided intramuscular steroid injection (Fig. 3) to bear with her symptoms prior to surgery.

There was an objective improvement in the patient's visual analogue scale (VAS) score after the intramuscular steroid injection. Her pre-procedure VAS score was 4-5 on sitting and 9 on extending the leg. Post-procedure VAS score improved to 2 on sitting and 5 on extending the leg. She underwent left hip ischiofemoral impingement decompression with lesser trochanter resection 19 months after her initial presentation, with subsequent symptomatic improvement.

\section{DISCUSSION}

Ischiofemoral impingement is an uncommon cause of hip, groin or posterior thigh pain. It is caused by narrowing of the shortest distance between the lateral cortex of the ischial tuberosity and the medial cortex of the lesser trochanter of the femur, which is known as the ischiofemoral space. The pain arises as a result of compression of soft tissues between the two bony prominences, in particular the quadratus femoris muscle. Another parameter that is referenced in this article is the quadratus femoris space, which is defined as the shortest distance for passage of the quadratus femoris muscle. It is measured from the superolateral surface of the hamstring tendons to the posteromedial surface of the iliopsoas tendon. The quadratus femoris muscle is an external rotator of the hip. It arises from the superolateral aspect of the ischial tuberosity, just anterior to the semimembranosus, and inserts into the quadrate tubercle and the intertrochanteric crest of the femur, occupying an approximately $2-\mathrm{cm}$ width between the ischium and the lesser trochanter. The anterior border of the quadratus femoris is formed by the obturator externus. The quadratus femoris muscle is bordered posteriorly by the sciatic nerve and insertion of the common hamstring tendon.

Ischiofemoral impingement is typically seen in middle-aged and elderly women, while it is relatively uncommon in male

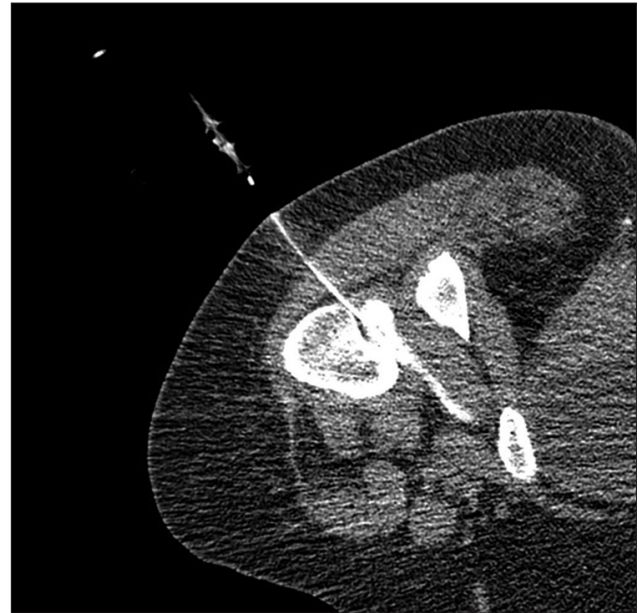

Fig. 3 Axial CT image of the left hip, with patient in the prone position, shows CT-guided injection of steroid and iodinated contrast into the right quadratus femoris muscle.

patients. There is bilateral hip involvement in $25 \%-40 \%$ of cases. ${ }^{(1,2)}$ Patients typically present with hip or groin pain on the affected side, which may radiate to the ipsilateral posterior thigh and knee. The patient may also experience snapping, locking or crepitus of the hip. ${ }^{(1,3,4)}$ Lower back pain may also be present. The patient may report a history of trauma. On physical examination, there is usually a tender range of motion of the affected hip, especially with adduction, extension and external rotation. ${ }^{(2,3,5)}$

When commoner causes for hip pain, such as hip fractures, have been excluded on plain radiography, MR imaging should be considered for further evaluation of the affected hip joint. However, there are subtle findings on plain radiography that may be able to point to the correct diagnosis. In patients with ischiofemoral impingement, plain radiographs usually appear normal but may show increased proximity of the ischium and lesser trochanter. In more severe cases, subcortical cysts and sclerosis involving the adjacent bony prominences may be detected. ${ }^{(6)}$ A study by Park et $\mathrm{al}^{(7)}$ suggests that plain pelvic radiographs may be used as a screening tool for ischiofemoral impingement in patients with chronic hip pain, using ischiofemoral distance cut-off points of $\leq 2 \mathrm{~cm}$ and $\leq 1.9 \mathrm{~cm}$ for supine and standing views, respectively, to denote narrowing of the ischiofemoral distance. In the patient's standing pelvic radiograph, the left ischiofemoral distance was $1.8 \mathrm{~cm}$ and the right ischiofemoral distance was $2.5 \mathrm{~cm}$ (Fig. 4).

In our case, the possibility of ischiofemoral impingement could be inferred from a plain radiograph given the accompanying clinical symptoms, but MR imaging remains the gold standard for definitive diagnosis. This is due to several limitations with the interpretation of plain radiographs. Firstly, the positioning of the patient has to be optimised - a true anteroposterior projection is required for accurate assessment of ischiofemoral distances. Also, the patient should avoid external rotation of the hip joints, as this may lead to overestimation of the ischiofemoral distances. Furthermore, the isolated narrowing of ischiofemoral distances in the absence of demonstrable interposing muscle oedema is non-specific. Hence, MR imaging is required to demonstrate the muscle oedema and clinch the diagnosis. 


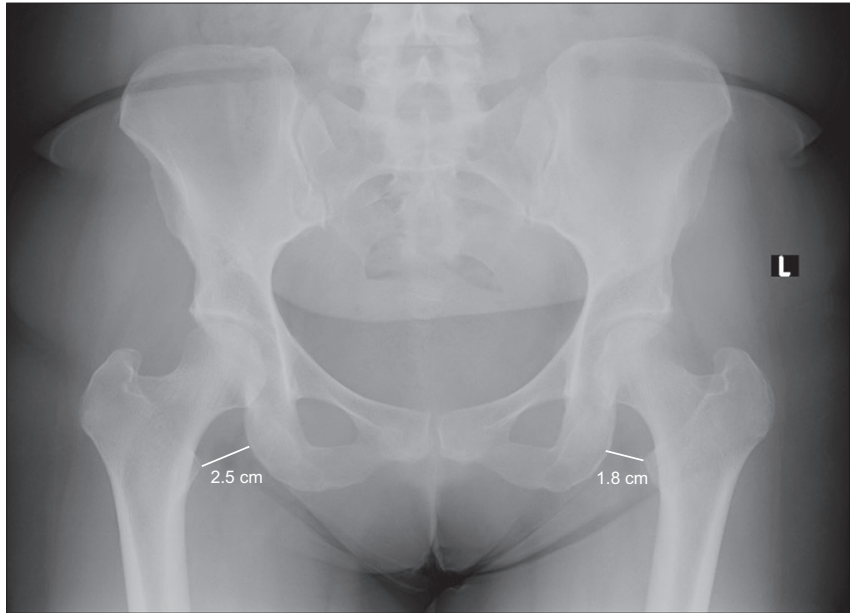

Fig. 4 Frontal radiograph of the patient's pelvis shows narrowing of the ischiofemoral distance, with the left ischiofemoral distance measuring $1.8 \mathrm{~cm}$ and the right ischiofemoral distance measuring $2.5 \mathrm{~cm}$.
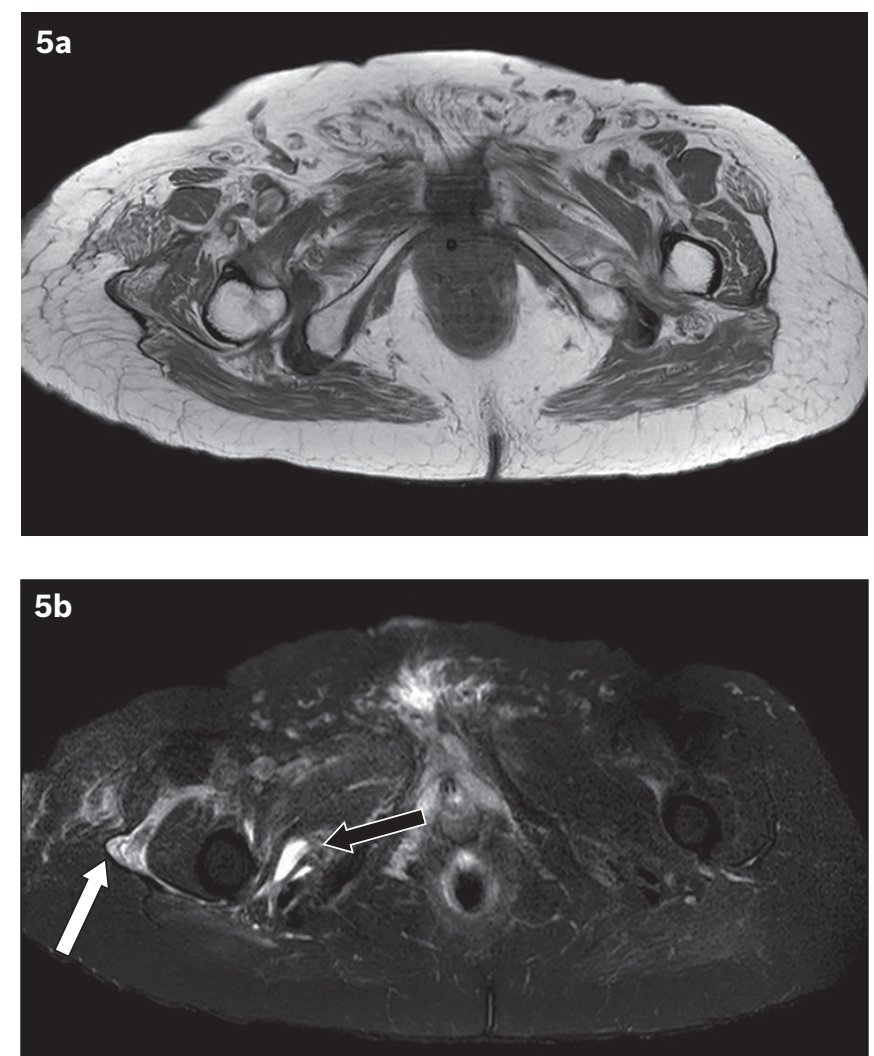

Fig. 5 An 82-year-old Chinese man with right ischiofemoral impingement. (a) Axial T1-weighted MR image shows narrowing of the right ischiofemora space. (b) Axial spectral attenuated inversion recovery MR image shows increased signal intensity in the right quadratus femoris muscle, in keeping with oedema (black arrow). Right greater trochanteric bursitis (white arrow) is also seen.

MR imaging features of ischiofemoral impingement include the following: narrowing of the ischiofemoral and/or quadratus femoris space, and oedema involving the quadratus femoris muscle at the site of maximal impingement. Other imaging features include complications such as a partial tear of the quadratus femoris muscle, tear or inflammation of the hamstring tendon origin, inflammation of the iliopsoas tendon, fluid collections or bursa-like formations (Fig. 5), sciatic nerve involvement, bone marrow oedema, and sclerosis or cystic change of the ischium
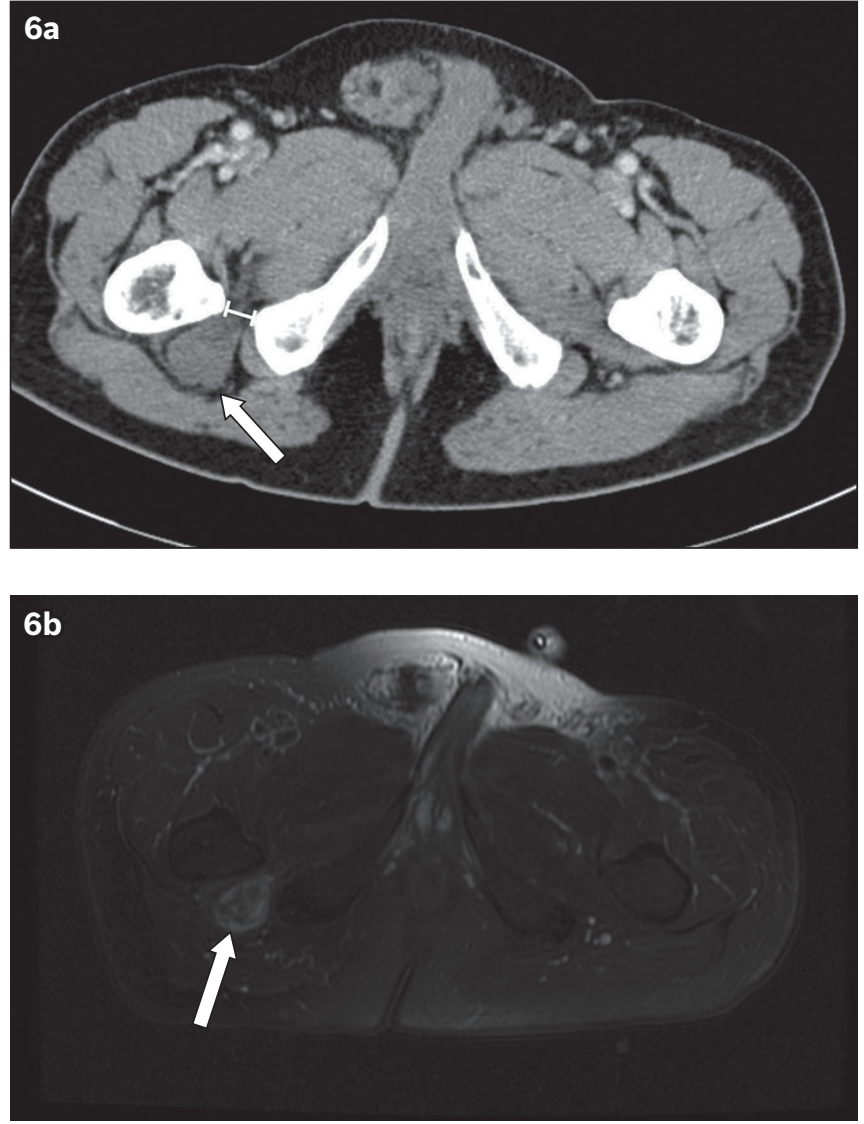

Fig. 6 A 66-year-old asymptomatic Chinese man with an incidental finding of a narrowed right ischiofemoral space and a right sciatic peripheral nerve sheath tumour. (a) Contrast-enhanced axial CT image of the pelvis shows a narrowed right ischiofemoral space (double-headed line) and a soft tissue mass along the course of the right sciatic nerve (arrow). (b) Axial T2 fat-saturated MR image of the pelvis shows the right sciatic nerve sheath tumour demonstrating heterogeneous, predominantly high signal intensity (arrow).

and lesser trochanter. In chronic cases, there can be atrophy and fatty replacement of the involved muscles. Other entities may coexist with ischiofemoral impingement and be demonstrated concurrently (Fig. 6).

According to a meta-analysis by Singer et al, ${ }^{(8)} \mathrm{MR}$ imaging demonstrating an ischiofemoral space cut-off value $\leq 15 \mathrm{~mm}$ has $77 \%$ sensitivity and $81 \%$ specificity for ischiofemoral impingement. A quadratus femoris space cut-off value $\leq 10 \mathrm{~mm}$ has $79 \%$ sensitivity and $74 \%$ specificity for the condition. Tosun et $\mathrm{al}^{(2)}$ studied 70 hips in 50 patients with hip pain and oedema of the quadratus femoris muscle to evaluate the utility of several MR imaging parameters to diagnose ischiofemoral impingement. The ischiofemoral and quadratus femoris spaces of the patient group were significantly narrower than those of the controls, with a mean ischiofemoral space of $13 \mathrm{~mm}$ in patients with ischiofemoral impingement versus $23 \mathrm{~mm}$ in the control group. The mean quadratus femoris space was $7 \mathrm{~mm}$ in the patients and $12 \mathrm{~mm}$ in the controls.

Ischiofemoral impingement is often idiopathic, although there are several recognised aetiologies. These can be broadly divided into congenital and acquired causes. Congenital aetiologies include female gender ${ }^{(1,2)}$ (female pelvises confer a relative predilection due to everted ischial tuberosities) and coxa valga. 
Acquired aetiologies include osteoarthritis, enthesopathy from the hamstring origin, osteochondroma, prior fracture resulting in prominent lesser trochanter or enlarged ischium, displaced intertrochanteric fracture, valgus intertrochanteric osteotomy and reduction of horizontal offset in hip arthroplasty.

Treatment is either conservative or invasive. Conservative management with analgesia and physiotherapy can provide some benefit. If this fails, image-guided intramuscular steroid injections may be useful, having provided complete but transient symptomatic relief in a study by Wilson and Keene. ${ }^{(9)}$ The definitive treatment is decompressive surgery with resection of the lesser trochanter.

In summary, ischiofemoral impingement is an uncommon cause of hip pain that usually occurs in middle-aged and elderly women. Compression of the quadratus femoris muscle with resultant muscle oedema leads to pain. In more severe cases, the surrounding soft tissue, tendons and even the sciatic nerve may be affected, causing sciatica. Plain radiographs may give a clue to the diagnosis, but MR imaging is usually required for a definitive diagnosis. This entity is one to consider for patients with hip pain in whom an ipsilateral hip fracture has been excluded.
ABSTRACT A 48-year-old woman presented with progressive left hip pain over six months, along with left lower limb weakness for two weeks. Magnetic resonance imaging of the left hip showed narrowing of the left ischiofemoral space, oedema of the left quadratus femoris muscle and left sciatic nerve, and mild bone marrow oedema of the left ischial tuberosity. The diagnosis of left ischiofemoral impingement syndrome was made. The imaging features of ischiofemoral impingement syndrome and the associated aetiologies and complications are discussed.

Keywords: hip pain, groin pain, ischiofemoral impingement syndrome, quadratus femoris

\section{REFERENCES}

1. Torriani M, Souto SC, Thomas BJ, Ouellette H, Bredella MA. Ischiofemoral impingement syndrome: an entity with hip pain and abnormalities of the quadratus femoris muscle. AJR Am J Roentgenol 2009; 193:186-90.

2. Tosun $\mathrm{O}$, Algin $\mathrm{O}$, Yalcin N, et al. Ischiofemoral impingement: evaluation with new MRI parameters and assessment of their reliability. Skeletal Radiol 2012; 41:575-87.

3. Tosun Ö, Çay N, Bozkurt M, Arslan H. Ischiofemoral impingement in an 11-year-old girl. Diagn Interv Radiol 2012; 18:571-3.

4. Ali AM, Whitwell D, Ostlere SJ. Case report: imaging and surgical treatment of a snapping hip due to ischiofemoral impingement. Skeletal Radiol 2011; 40:653-6.

5. Stafford GH, Villar RN. Ischiofemoral impingement. J Bone Joint Surg Br 2011; 93:1300-2

6. Patti JW, Ouellette H, Bredella MA, Torriani M. Impingement of lesser trochanter on ischium as a potential cause for hip pain. Skeletal Radiol 2008; 37:939-41.

7. Park S, Lee HY, Cuong PM, et al. Supine versus standing radiographs for detecting ischiofemoral impingement: a propensity score-matched analysis. AJR Am J Roentgenol 2016; 206:1253-63.

8. Singer AD, Subhawong TK, Jose J, Tresley J, Clifford PD. Ischiofemoral impingement syndrome: a meta-analysis. Skeletal Radiol 2015; 44:831-7.

9. Wilson MD, Keene JS. Treatment of ischiofemoral impingement: results of diagnostic injections and arthroscopic resection of the lesser trochanter. J Hip Preserv Surg 2016; 3:146-53. 


\section{SINGAPORE MEDICAL COUNCIL CATEGORY 3B CME PROGRAMME} (Code SMJ 201907B)

Question 1. Regarding the clinical presentation of a patient with ischiofemoral impingement syndrome:

(a) Lower back pain precludes the diagnosis of ischiofemoral impingement syndrome.

(b) Patients typically present with hip or groin pain, worsened by movement of the affected hip.

(c) The majority of patients present with bilateral hip involvement.

(d) Tender range of motion involving the affected hip is exacerbated by adduction and external rotation.

Question 2. Regarding the quadratus femoris muscle:

(a) It is an internal rotator of the hip.

(b) It arises from the ischial tuberosity and inserts into the quadrate tubercle and the intertrochanteric crest of the femur.

(c) It is in close relation to the sciatic nerve.

(d) It is bordered anteriorly by the insertion of the common hamstring tendon.

Question 3. Regarding the radiological diagnosis of ischiofemoral impingement syndrome:

(a) Accurate diagnosis can usually be made on plain radiography alone, by detecting reduction in the ischiofemoral distances.

(b) Subcortical cysts and sclerosis involving the ischial tuberosity and lesser trochanter of the femur are detectable on plain radiographs.

(c) Magnetic resonance (MR) imaging allows for the detection of muscle oedema.

(d) Computed tomography is often sufficient for diagnosis.

Question 4. The following imaging findings may be seen in MR evaluation of a patient with ischiofemoral impingement syndrome:

(a) Narrowing of the ischiofemoral and/or quadratus femoris space.

(b) Oedema involving the quadratus femoris muscle.

(c) Tear or inflammation of the obturator externus muscle.

(d) Fluid collections or bursa-like formation.

Question 5. Predisposing conditions for the development of ischiofemoral impingement syndrome include:

(a) Coxa valga.

(b) Prior displaced intertrochanteric fracture of the femur.

(c) Valgus intertrochanteric osteotomy.

(d) Prior injury resulting in prominence of the greater trochanter.

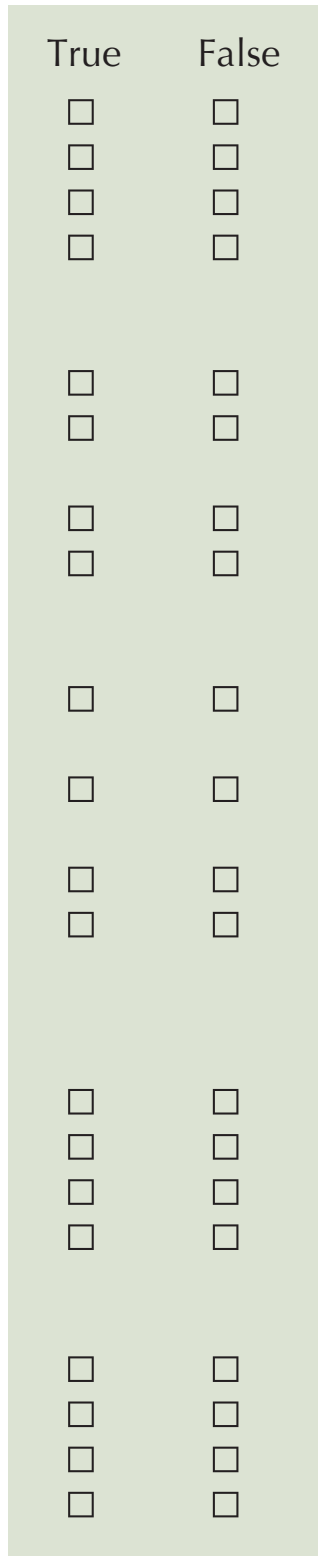

\section{Doctor's particulars:}

Name in full:

MCR no.:

Specialty:

Email:

\footnotetext{
SUBMISSION INSTRUCTIONS:

Visit the SMJ website: http://www.smj.org.sg/current-issue and select the appropriate quiz. You will be redirected to the SMA login page.

For SMA member: (1) Log in with your username and password (if you do not know your password, please click on 'Forgot your password?'). (2) Select your answers for each quiz and click 'Submit'.

For non-SMA member: : (1) Create an SMJ CME account, or log in with your SMJ CME username and password (for returning users). (2) Make payment of SGD 21.40 (inclusive of $7 \%$ GST) via PayPal to access this month's quizzes. (3) Select your answers for each quiz and click 'Submit'.

RESULTS:

(1) Answers will be published online in the SMJ September 2019 issue. (2) The MCR numbers of successful candidates will be posted online at the SMJ website by 10 September 2019. (3) Passing mark is $60 \%$. No mark will be deducted for incorrect answers. (4) The SMJ editorial office will submit the list of successful candidates to the Singapore Medical Council. (5) One CME point is awarded for successful candidates. (6) SMC credits CME points according to the month of publication of the CME article (i.e. points awarded for a quiz published in the December 2017 issue will be credited for the month of December 2017, even if the deadline is in January 2018).

Deadline for submission (July 2019 SMJ 3B CME programme): 12 noon, 3 September 2019.
} 